\title{
Active Polycondensation of Diesters Having Heterocyclic Nuclei
}

\author{
Naoya Ogata and Kiyoshi Shimamura \\ Department of Chemistry, Sophia University, \\ 7 Kioi-Cho, Chiyoda-Ku, Tokyo 102, Japan.
}

(Received June 21, 1974)

\begin{abstract}
Diesters having heterocyclic nuclei, such as furan, thiophene, pyridine, and pyrazine, were found to undergo a polycondensation reaction with hexamethylenediamine in solutions under mild conditions. The reactivity enhancement of the diesters due to the introduction of heterocyclic nuclei could be arranged in the following order: pyrazine $>$ pyridine $>$ furan $>$ thiophene. The enhancement effect of these heterocyclic nuclei was not directly related to the basicities of the diesters and it might be ascribed to the same reason as the enhancement described in previous papers.
\end{abstract}

KEY WORDS Polycondensation / Diester / Heterocyclic Nuclei /

Pyrazine / Hexamethylenediamine /

It was reported in previous papers ${ }^{1,2}$ that diesters having hetero atoms such as oxygen, sulfur, or nitrogen atoms at positions $\alpha$ or $\beta$ to the carbonyl group of the diesters were easily subjected to polycondensation reactions with aliphatic diamines under such mild conditions that the polycondensation reaction proceeded at room temperature in methanol solution. Aromatic diesters having $\beta$-ether group which were synthesized from salicylate also had a high reactivity toward the polycondensation with diamines. ${ }^{3}$ This enhancement effect of these hetero atoms on the polycondensation was presumed to be due to an inductive effect of these hetero atoms and also to an interaction of the hetero atoms with the amine approaching near the carbonyl group of the ester.

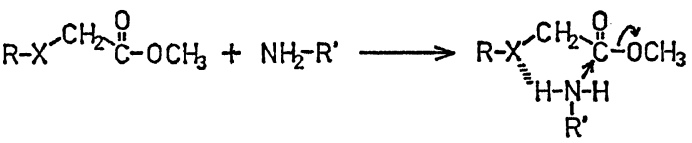

$$
\begin{aligned}
& \longrightarrow \mathrm{R}-\mathrm{X}-\mathrm{CH}_{2} \mathrm{CONH}-\mathrm{R}^{\prime} \\
& +\mathrm{CH}_{3} \mathrm{OH}
\end{aligned}
$$

This paper deals with further extensive studies on the reactivity enhancement of diesters toward amines which was caused by the introduction of hetero atoms into the skelton structure of the diesters. Several diesters having heterocyclic nuclei such as furan, thiophene, or pyridine were synthesized and their polycondensation with hexamethylenediamine was carried out in solution or in bulk under mild conditions.

\section{EXPERIMENTAL}

Syntheses of Heterocyclic Monomers

The following seven heterocyclic diesters were synthesized:
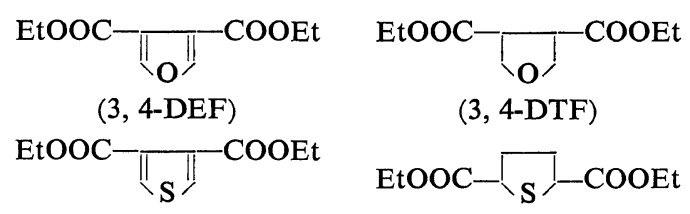

(3, 4-DTF)

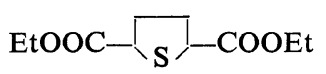

(2, 5-DTT)

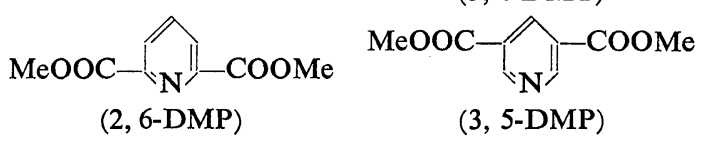<smiles>COC(=O)C1C2CCC(CC2)C1C(=O)OC</smiles>

(3, 6-DPR)

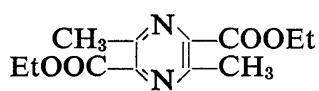


3, 4-Dicarboethoxy furan $(3,4-D E F)$ was synthesized by the method of Kornfeld ${ }^{4}$ from diethyl succinate and ethyl formate through the following route:

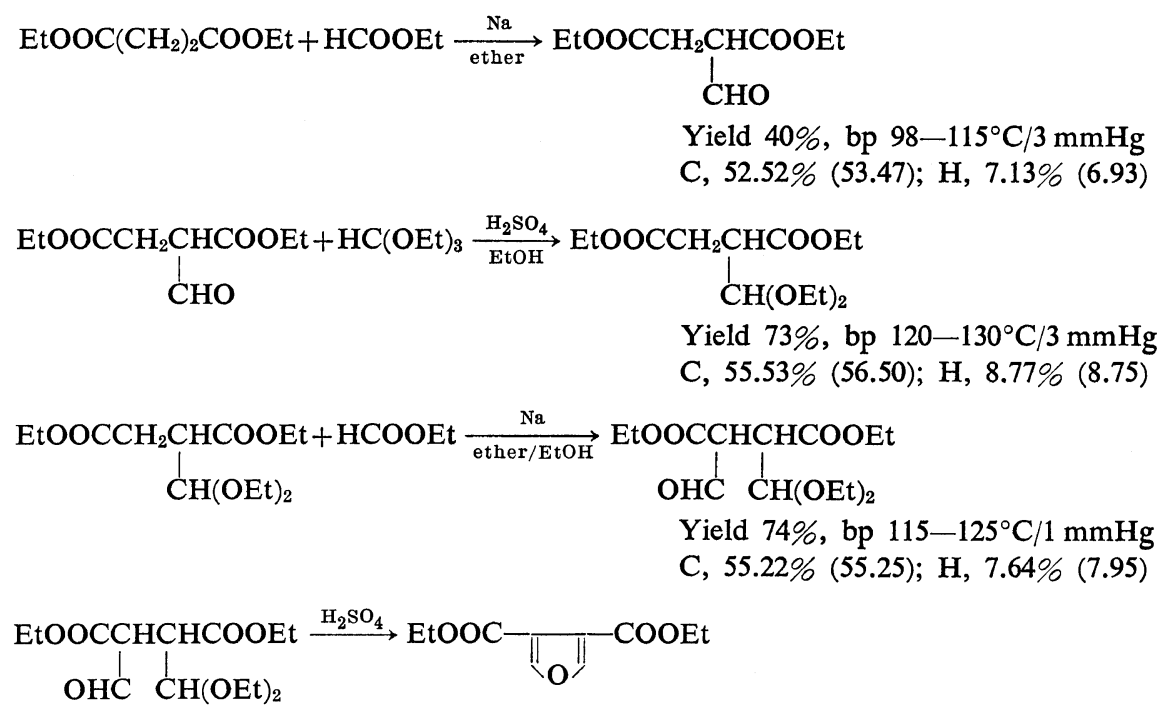

Yield $35 \%$, bp $112-115^{\circ} \mathrm{C} / 3 \mathrm{mmHg}$ C, $55.95 \%$ (56.60), H, 5.95\% (5.70)

3, 4-DEF obtained was purified by repeated distillation under reduced pressures.

Hydrogenation of 3,4-DEF was carried out in dioxane in the presence of Raney $\mathrm{Ni}$ under a hydrogen pressure of $150 \mathrm{~atm}$ at $200^{\circ} \mathrm{C}$ until the hydrogen absorption had stopped for $3 \mathrm{hr} .3$, 4Dicarboethoxy tetrahydrofuran (3, 4-DTF), which was a trans form, was purified by vacuum distillation.

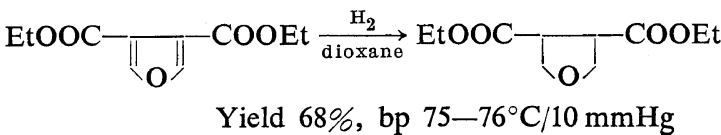

3, 4-Dicarboethoxy thiophene (3, 4-DMT) was synthesized from diethyl-1-formyl-2-diethoxymethyl succinate in toluene in the presence of phosphorus pentasulfide. ${ }^{4}$ 3, 4-DMT was purified by repeated recrystallization from ethanol and water.<smiles>CCOC(=O)C(CC(C=O)C(C(=O)OCC)C(C=O)C(=O)OCC)C(=O)OCC</smiles>

Yield $24 \%, \mathrm{mp} 58-59^{\circ} \mathrm{C}$ (lit. $\mathrm{mp} 58^{\circ} \mathrm{C}$ )

Since the hydrogenation of thiophene is very difficult, 1, 4-dicarboethoxy tetrahydrothiophene
(1, 4-DTT) was synthesized by the reaction of 1, 4-dibromo diethyl adipate with sodium sulfide in ethanol at room temperature for 10 days. 1, 4-DTT was purified by vacuum distillation.

$$
\begin{aligned}
& \text { EtOOCCH}\left(\mathrm{CH}_{2}\right)_{2} \mathrm{CHCOOEt} \\
& \stackrel{\mathrm{Br}}{\mathrm{Br}} \\
& \stackrel{\mathrm{Na} 2 \mathrm{~S}}{\mathrm{Yield}} 33 \% \text {, bp } 50-52^{\circ} \mathrm{C} / 3 \mathrm{mmHg}
\end{aligned}
$$

2,6- or 3,5-Dicarbomethoxy pyridines (2, 6or $3,5-\mathrm{DMP}$ ) were synthesized by the oxidation ${ }^{5}$ of 2,6- or 3,5-lutidines with potassium permanganate in aqueous solution, followed by esterification with methanol. 2,6- and 3, 5-DMP were purified by recrystallization from ethanol.

$$
\stackrel{\mathrm{MeOH}}{\longrightarrow} \mathrm{MeOOC} \stackrel{\mathrm{COOOCO}}{\longrightarrow}
$$

Hydrogenation of 2,6-DMP was carried out in dioxane at $130^{\circ} \mathrm{C}$ under a hydrogen pressure of $120 \mathrm{~atm}$ in the presence of Raney $\mathrm{Ni}$ to obtain 2, 6-dicarbomethoxy piperidine (2, 6-DPP). 


\section{N. Ogata and K. Shimamura}

2, 6-DPP was purified by vacuum distillation.

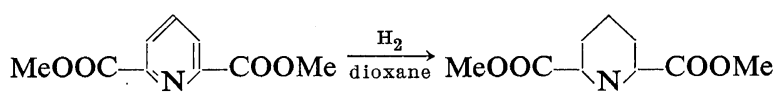

Yield $72 \%, \mathrm{mp} 88-89^{\circ} \mathrm{C}$ bp $122-124^{\circ} \mathrm{C} / 3 \mathrm{mmHg}$

3, 6-Dicarboethoxy-2, 5-dimethyl pyrazine (3,6-DPR) was synthesized according to the method of Iida $^{6}$ from ethyl acetoacetate and sodium nitrite in acetic acid.

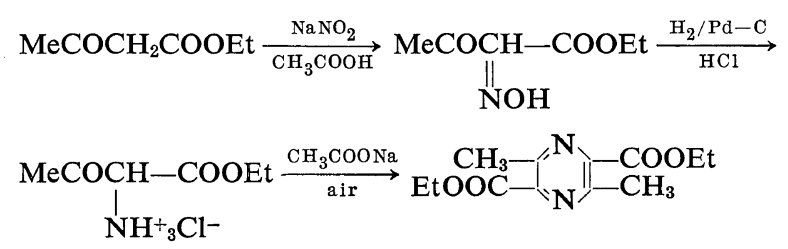

Yield $32 \%, \mathrm{mp} 88^{\circ} \mathrm{C}$ (lit. $\mathrm{mp} 88^{\circ} \mathrm{C}$ )

In Table I are summarized the properties and the elemental analyses of these heterocyclic diesters.

$N$-Methylpyridinium salts of pyridine esters were obtained by reacting the corresponding pyridine esters with an equimolar amount of methyl iodide in methanol.

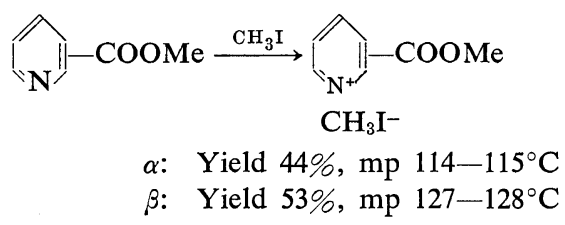

Table I. Properties and analytical results of heterocyclic diesters

\begin{tabular}{|c|c|c|c|c|c|}
\hline Diesters & Abbreviation & $\mathrm{bp}$ or $\mathrm{mp}$ & $\mathrm{C}, \%$ & $\mathbf{H}, \%$ & $\mathrm{~N}, \%$ \\
\hline EtOOC $-\|_{O}^{\|}-$COOEt & 2, 3-DEF & $125-127 / 6 \mathrm{mmHg}$ & $\begin{array}{c}56.15 \\
(56.60)\end{array}$ & $\begin{array}{l}5.97 \\
(5.70)^{\mathrm{a}}\end{array}$ & - \\
\hline EtOOC- & 2, 3-DTF & $75-76 / 10 \mathrm{mmHg}$ & $\begin{array}{l}55.40 \\
(55.55)\end{array}$ & $\begin{array}{c}7.32 \\
(7.40)\end{array}$ & - \\
\hline $\mathrm{MeOOC}-\left\|_{S}\right\|^{-C O O M e}$ & 2, 3-DMT & {$[58-59]$} & $\begin{array}{l}48.27 \\
(48.00)\end{array}$ & $\begin{array}{c}3.90 \\
(4.00)\end{array}$ & - \\
\hline EtOOC- & 1, 4-DTT & $128-130 / 5 \mathrm{mmHg}$ & $\begin{array}{c}51.88 \\
(51.72)\end{array}$ & $\begin{array}{c}7.03 \\
(6.90)\end{array}$ & - \\
\hline -COOMe & 2, 6-DMP & {$[125-127]$} & $\begin{array}{c}55.28 \\
(54.80)\end{array}$ & $\begin{array}{c}4.68 \\
(4.57)\end{array}$ & $\begin{array}{c}7.27 \\
(7.12)\end{array}$ \\
\hline COOMe & 3,5-DMP & [88-89] & $\begin{array}{l}54.85 \\
(54.80)\end{array}$ & $\begin{array}{c}4.61 \\
(4.57)\end{array}$ & $\begin{array}{l}7.21 \\
(7.12)\end{array}$ \\
\hline$\widehat{N}_{\mathrm{H}}^{\prime}-\mathrm{COOMe}$ & 2, 6-DPP & $\begin{array}{l}{[88-89]} \\
122-124 / 3 \mathrm{mmHg}\end{array}$ & $\begin{array}{c}53.91 \\
(53.73)\end{array}$ & $\begin{array}{c}7.58 \\
(7.46)\end{array}$ & $\begin{array}{l}6.82 \\
(6.96)\end{array}$ \\
\hline $\begin{array}{c}\mathrm{CH}_{3}-\mathrm{N}_{\mathrm{N}}-\mathrm{COOEt} \\
\mathrm{EtOOC}-\mathrm{CH}_{3}\end{array}$ & 3, 6-DPR & {$[88]^{\mathrm{b}}$} & - & - & - \\
\hline
\end{tabular}

a Calculated values.

b lit. $\mathrm{mp} 88^{\circ} \mathrm{C}$. 
Active Polycondensation

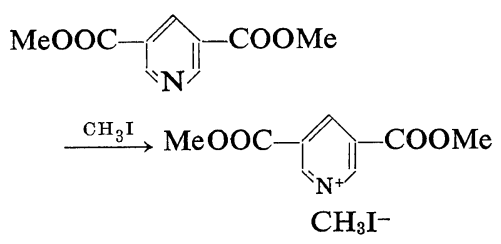

( $N$-methyl-3, 5-DMP iodide)

Yield $45 \%$, mp $197-199^{\circ} \mathrm{C}$

Nicotinate $N$-oxide was obtained by the oxidation of methyl nicotinate with hydrogen peroxide in acetic acid.

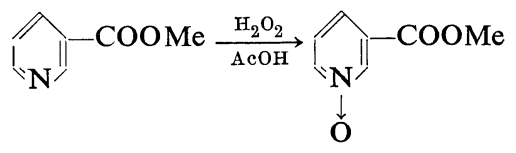

Yield $25 \%, \mathrm{mp} 94-95^{\circ} \mathrm{C}$

\section{Polycondensation Reaction}

Equimolar amounts of diesters and hexamthylenediamine (HMD) were dissolved in various solvents in a given concentration; the solutions were kept at a constant temperature. The rate of the polycondensation reaction was followed either by the amount of residual amino group in the solution, which was measured by titration with $0.1-\mathrm{N} \mathrm{HCl}$, or by the amount of liberated alcohol, which was determined by gaschromatography using a poly(ethylene glycol) column.

The polymers formed were precipitated by pouring the solution into excess acetone, followed by repeated washing with acetone and drying. The melting points of the resulting polymers were measured by a differential theormometer, using Rigakudenki model "Thermoflex". Solution viscosities of the polymers were measured in $m$-cresol at $30^{\circ} \mathrm{C}$.

\section{RESULTS AND DISCUSSION}

\section{Model Reaction}

Condensation reactions of monoesters containing pyridine or furan with hexamethylenediamine (HMD) were carried out in hexamethylenephosphoramide (HMPA) at $60^{\circ} \mathrm{C}$ in the HMD concentration of $0.4 \mathrm{~mol} / l$. Methyl benzoate was used as a standard monomer against which to compare the reactivity of the monoesters. Figure 1 indicates that the monoesters containing a pyridine nucleus are easily subjected to the

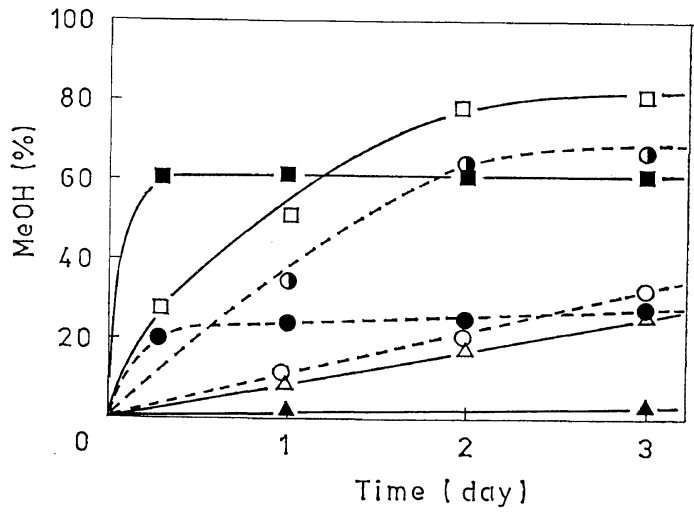

Figure 1. Condensation reactions of monoesters with $\mathrm{HMD}$ in $\mathrm{HMPA}$ at $60^{\circ} \mathrm{C}$ :

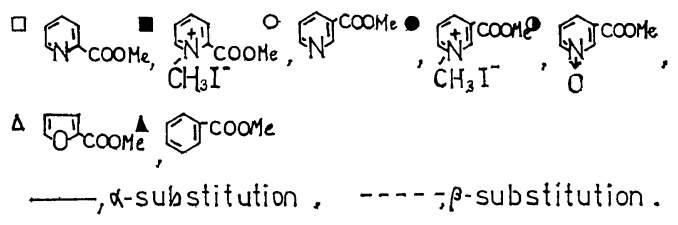

condensation reaction with HMD with a much faster rate than that of methyl benzoate. The reactivity of the monoester was influenced by the position of the ester group on the pyridine nucleus, that is, methyl picolinate ( $\alpha$-ester) reacted faster than methyl nicotinate ( $\beta$-ester). This reactivity order was consistent with the results of aliphatic diesters containing hetero atoms at positions $\alpha$ or $\beta$ to the ester carbonyl group. $^{2}$

When the pyridine nucleus was transformed into $N$-methylpyridinium or pyridine $N$-oxide structures, the condensation reaction took place with much faster rates than those of picolinate or nicotinate. However, $\mathrm{N}$-methylpyridinium esters reached an apparent equilibrium as soon as the condensation reaction started.

The high reactivity of esters containing a pyridine nucleus might be presumably ascribed to an autocatalytic effect of basic pyridine nitrogen. Since $\mathrm{p} K_{\mathrm{a}}$ values of methyl picolinate $(\alpha$-ester) and methyl nicotinate ( $\beta$-ester) are known ${ }^{7}$ to be 2.21 and 3.13, respectively, methyl nicotinate should have a higher reactivity toward amidation reaction than methyl picolinate if one would postulate that the basicity of the esters is a predominant factor for the reactivity enhance- 


\section{N. Ogata and K. Shimamura}

ment. Nevertheless, the condensation reaction of methyl picolinate proceeded faster than that of methyl nicotinate. Therefore, it is concluded that the reactivity enhancement of the pyridine esters is not only based on the basic character of pyridine nucleus, but also on other factors which must be considered predominant for the reactivity enhancement.

\section{Polycondensation Reaction}

Polycondensation of 3,4-DEF or 3,4-DTF with HMD was carried out in HMPA solution at $60^{\circ} \mathrm{C}$; the results are shown in Figure 2, which indicates that both monomers are easily subjected to polycondensation with faster rates than that of dimethylisophthalate (DMI) with

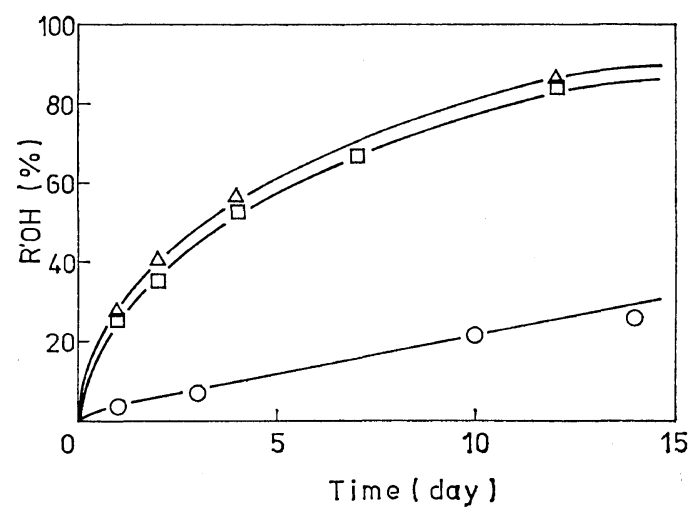

Figure 2. Polycondensation of diesters having furan rings with $\mathrm{HMD}$ at $60^{\circ} \mathrm{C}$, monomer concn $0.4 \mathrm{~mol} / l ; \triangle 3,4-\mathrm{DEF}, \square 3,4-\mathrm{DTF}, \bigcirc \mathrm{DMI}$.

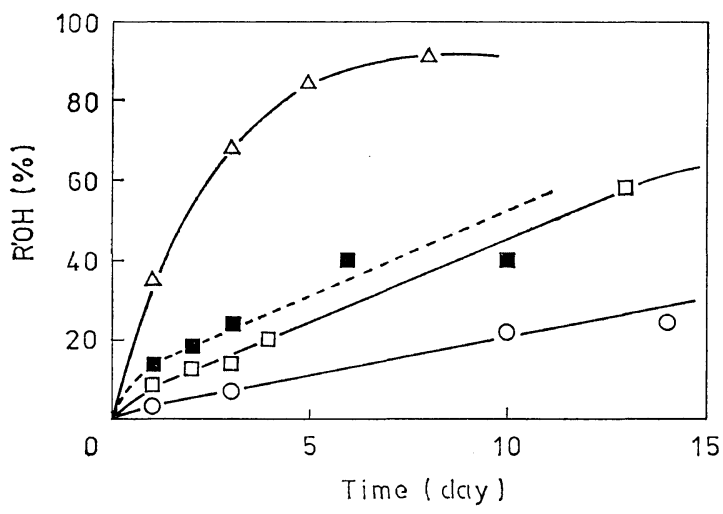

Figure 3. Polycondensation of diesters having thiophene rings with $\mathrm{HMD}$ at $60^{\circ} \mathrm{C}$, monomer concn $0.4 \mathrm{~mol} / l$ in HMPA; $\triangle 2,5$-DTT, $3,4-$ DMT in DMSO, $\square$ 3, 4-DMT, $\bigcirc$ DMI.
HMD. No significant difference in the polycondensation rates between furan and tetrahydrofuran structures was observed.

Figure 3 shows results of the polycondensation of 3,4-DMT or 2, 5-DTT with HMD in HMPA solution or dimethylsulfoxide (DMSO) solutions at $60^{\circ} \mathrm{C}$. It is seen in Figure 3 that both monomers had a higher reactivity than that of DMI and that 2,5-DTT reacted much faster than 3,4-DMT. This difference in reactivity between 2,5-DTT and 3,4-DMT is not only related to the character of the heterocyclic nuclei, but also to the substitution position of the ester group on the heterocyclic nuclei.

Figure 4 indicates the polycondensation reaction of diesters having azine rings in HMPA solution at $60^{\circ} \mathrm{C}$. It is seen in Figure 4 that the diesters having pyrazine or pyridine nuclei were easily subjected to the polycondensation with HMD. Among these azine diesters, 3, 6DPR had the highest reactivity. The polycondensation of 3,6-DPR was completed at $60^{\circ} \mathrm{C}$ within 3 days and a polyamide having an inherent viscosity of $0.4-0.5$ was obtained. The apparent overall rates of the polycondensation of these azine diesters could be arranged in the following order: 3, 6-DPR $>2,6$-DMP $>2,6$-DPP $>3,5$-DMP.

Since $\mathrm{p} K_{\mathrm{a}}^{-}$values of pyridine and pyrazine are known ${ }^{7}$ to be 5.17 and 0.6 , respectively, pyridine diesters are presumed to have a much stronger

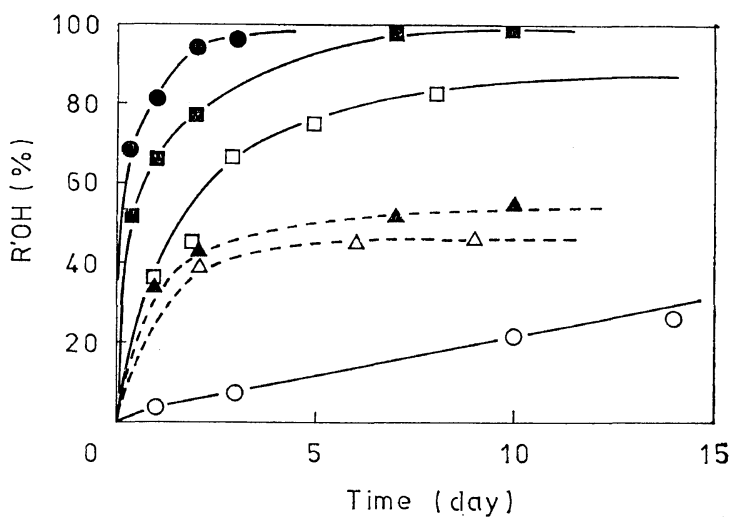

Figure 4. Polycondensation of diesters having azine rings with $\mathrm{HMD}$ at $60^{\circ} \mathrm{C}$, monomer concn $0.4 \mathrm{~mol} / l$ in HMPA; $3,6-\mathrm{DPR}$, 2,6-DMP, $\square$ 2,6-DPP, $\triangle 3,5$-DMP, $\triangle N$-methyl-3, 5-DMP iodide, $\bigcirc \mathrm{DMI}$. 


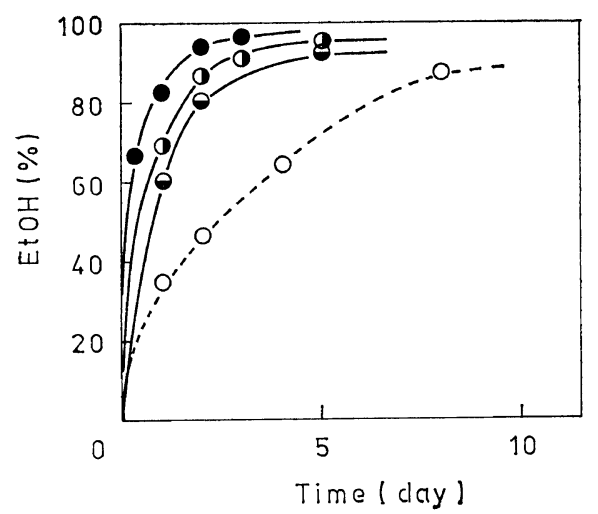

Figure 5. Polycondensation of 3,6-DPR with HMD in various solvents, monomer concn 0.4 $\mathrm{mol} / l, 60^{\circ} \mathrm{C}$; $\bigcirc \mathrm{HMPA}, \mathrm{CH}_{3} \mathrm{CN}, \bigcirc \mathrm{DMF}, \bigcirc$ Ethanol (conversion was determined by polymer yield).

basicity than 3,6-DPR if one would postulate that the basicity of the diesters is primarily determined by the basic character of the heterocyclic nuclei. Moreover, $N$-methylpyridinium salt of 3,5-DMP, which has a low basicity, had almost the same reactivity as that of 3,5DMP, as shown in Figure 4. Therefore, the basic character of azine rings is not a main factor in determining the rates of the polycondensation reaction. As indicated in Figure 4, 2, 6-DMP reacted faster with HMD than 3, 5-DMP and the position of the ester group also influenced the reactivity of these monomers.

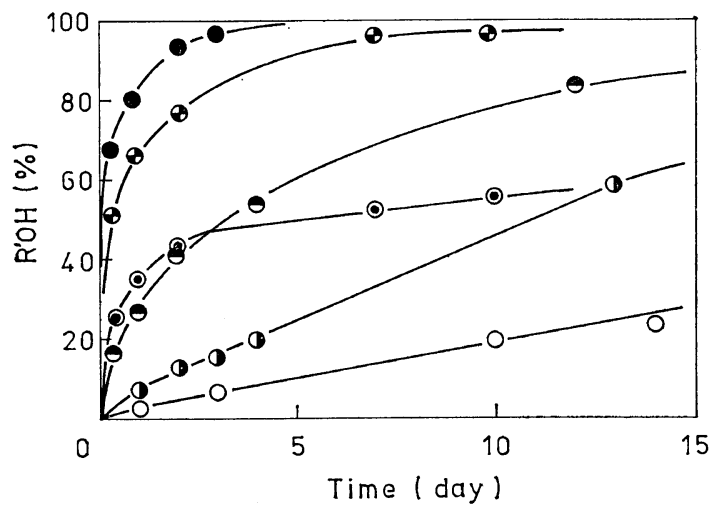

Figure 6. Polycondensation of various heterocyclic diesters with $\mathrm{HMD}$ at $60^{\circ} \mathrm{C}$, monomer concn 0.4 $\mathrm{mol} / \mathrm{l}$ in HMPA; 3, 6-DPR, 2, 6-DMP, $\odot 3,5$ DMP, 3,4-DEF, 3,4-DMT, $\bigcirc$ DMI.
Figure 5 indicates the polycondensation of 3, 6-DPR with HMD in various solvents. Among the solvents tested, HMPA was the best solvent in terms of the polycondensation rate. The polycondensation of 3,6-DPR with HMD proceeded even in ethanol.

Figure 6 summarizes results of the active polycondensation of various heterocyclic diesters with HMD. Apparent rate constants of the polycondensation reactions at the initial stage are collected in Table II, where the initial rate constants were calculated as for a second-order reaction. The initial rates of the polycondensation of these heterocyclic diesters increase in the following order:

$$
\begin{aligned}
& \mathrm{CH}_{3} \\
& >-\left\|_{0}\right\|>-\left\|_{S}\right\|>-
\end{aligned}
$$

The rate constant of 3,6-DPR having a pyrazine ring is 181 times as much as that of dimethyl isophthalate, as indicated in Table II. Apparent equilibrium constants of the polycondensation reaction are listed in Table II; they were calculated when the polycondensation reac-

Table II. Polycondensation of diester with HMD

$k^{, \mathrm{a}}$
$l \mathrm{~mol}^{-1} \mathrm{hr}^{-1}$


Table III. Polycondensation of $\mathrm{R}^{\prime \prime O O C}-\mathrm{R}-$ COOR" with $\mathrm{NH}_{2}-\mathrm{R}^{\prime}-\mathrm{NH}_{2}$

Solu-
bility

tion apparently reached equilibrium after several months.

Table III summarizes properties of the resulting polyamides which have heterocyclic nuclei. Most of these heterocylic polyamides were soluble in dimethylformamide (DMF) and polyamides having azine rings had a higher melting point than that of polyamides having furan or thiophene rings. A suggested explanation of the higher melting points of azine polyamides might be based on the strength of the hydrogen bonding between the nitrogen atom of azine rings and amide hydrogen.

The high reactivities of these heterocyclic diesters toward the polycondensation reaction can be presumably ascribed to the same effect as the reactivity enhancement of diesters having hetero atoms, as postulated in a previous paper. ${ }^{2}$ Further detailed studies on the reaction mechanism are still necessary and will be reported in the near future.

\section{REFERENCES}

1. N. Ogata, K. Sanui, and K. Okouchi, Polymer J., 5, 186 (1973).

2. N. Ogata, Y. Hosoda, and G. Suzuki, ibid., 6, 412 (1974).

3. N. Ogata and Y. Ishizaki, Kobunshi Ronbunshu, 31, 561 (1974).

4. E. C. Kornfeld and R. G. Jones, J. Org. Chem., 19, 1671 (1954).

5. G. Black, ibid., 14, 17 (1949).

6. H. Iida, K. Hayashida, and K. Takahashi, Yuki Gosei Kagaku Kyokaishi (J. Synth. Org. Chem. Japan), 31, 688 (1973).

7. A. Albert and J. N. Phillips, J. Chem. Soc., 1294 (1956). 